\title{
Optimizing Radio Resources for Heterogeneous QoS- aware OFDMA Networks using Semi-smart Antennas
}

\author{
Aini Li, Yue Liu, Laurie Cuthbert, Yue Gao \\ Queen Mary University of London \\ Electronic Engineering and Computer Science \\ London, UK \\ \{a.li\} \{yue.liu\} \{laurie.cuthbert\}\{yue.gao\}@qmul.ac.uk
}

\author{
Yapeng Wang \\ MPI-QMUL Information Systems Research Center (ISRC) \\ Macao SAR \\ yapeng.wang@qmul.ac.uk
}

\begin{abstract}
The need to consider the Quality of Service (QoS) requirements of individual users and the business strategies of different operators is gaining widespread acceptance in resource allocation. OFDMA is a suitable technology for delivering very high data rate services because of its inherent ability to remove intra-cell interference. However, cell-edge users will experience high interference from neighboring cells. This paper proposes a novel radio resource management scheme in multi-cell OFDMA spectrum sharing networks to achieve the business and QoS objectives. Semi-smart antennas are employed to mitigate the cochannel interference, especially for cell-edge users, and to improve energy utilization for the overall networks. A system level simulation is implemented and the results show the proposed scheme improves the qualified user number in different load networks within a satisfactory system throughput.
\end{abstract}

Keywords-QoS, business strategies, OFDMA, semi-smart antennas, resource management

\section{INTRODUCTION}

Orthogonal Frequency Division Multiple Access (OFDMA) has been recognised as one of the most viable technologies for future high-speed wireless networks. Although OFDMA is free from frequency-selective fading and offers some freedom in radio resource management (RRM) [1], there are still challenges to provide QoS to a large number of users with the limited frequency spectrum, the main one being inter-cell interference since neighboring cells may use the same subcarriers (SCs) [2].

However, most previous research in RRM for OFDMA networks has concentrated on the maximization of system throughput [3]-[11] and minimization of transmission power [12][13]. However, users are only concerned with their QoS, so it is necessary for the network operators to allocate resources according to each user's QoS requirement. Without that consideration it would be possible for (i) a fair allocation method to share the resources so fairly that none gets the desired QoS or (ii) a greedy method that gives a few users excessive resources while others can barely transmit [14].

Based on these considerations, the concepts of qualified users, qualified throughput and spectrum sharing network were proposed for QoS-aware resource allocation in [14] and [15]. Qualified user $(Q U)$ is that whose desired QoS is achieved and qualified throughput is the throughput of qualified users.
Spectrum sharing network is a heterogeneous OFDMA network with different users sharing the spectrum, with different classes of users subject to different rules. This can be thought of as a cognitive radio network but one where the criteria on which users get priority on spectrum are much more complex. The benefits of that approach were demonstrated in [14][15].

However, it is clear that the challenge in allocating resources in any OFDMA network is in serving cell-edge users. The research in this paper addresses that cell-edge problem, in the context of the heterogeneous mix of users, for instance primary user (PU) and secondary user (SU), by optimizing the shape of the cells, namely the cell radio coverage to fit the demands of the user distribution. In this research, PUs have a higher priority to obtain SC resource and SUs will only be served by their operator if there are spare resources after delivering the PUs required QoS. The simulation results show that significant improvement in the number of users meeting their QoS requirement can be achieved.

\section{SYSTEM MODEL}

\section{A. Network Model}

An OFDMA multi-cell network serving users with heterogeneous QoS requirements is considered here. The network applies a 7-cell wrap around model with 3 sectors per cell. Users can be distributed in different ways depending on the scenario.

The overall approach is to compare the number of qualified users with and without the antenna patterns being optimized.

\section{B. Antenna Model and Coverage}

The semi-smart antennas at the base stations (BSs) each consists of three sectors with a 2-element array antenna for each sector. The radiation pattern of the semi-smart antenna is shown in Figure 1 with each element covering 60 degree. The antenna gains along six directions can be represented by $\boldsymbol{g}_{1}, \boldsymbol{g}_{2}, \ldots, \boldsymbol{g}_{\boldsymbol{6}}$. The value of $\boldsymbol{g}_{\boldsymbol{j}}(j=1,2 \ldots 6)$ is adjustable and can be optimized to suit the particular user distribution.

It would be possible to use a more complex set of antennas and control the phase as well as the gain, but the approach adopted here is simple but more realistic in practice. 


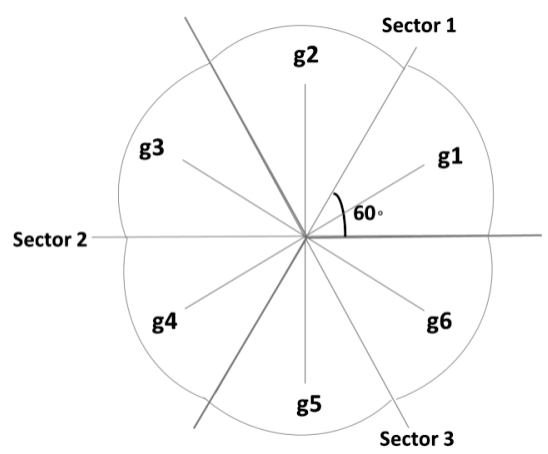

Figure 1 Antenna segments with variable gain

The semi-smart antennas allow the cell radius to become adaptive instead of static, leading to a dynamic cell boundary and flexible cell coverage, shown in Figure 2. Dashed cells represent the original uniform cellular network; solid cells represent the network employing the semi-smart system and showing adaptation to the high traffic load present at the central cell.

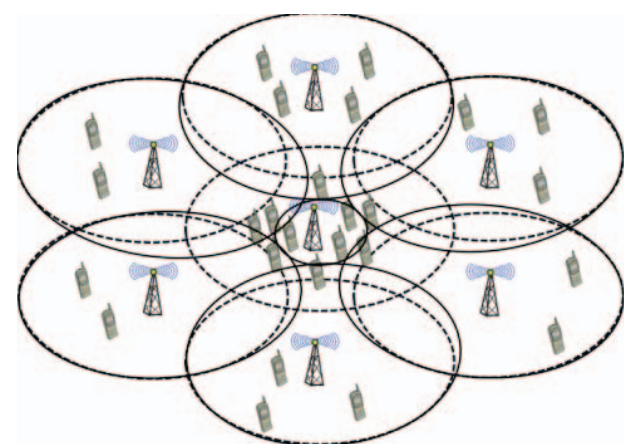

Figure 2 Basic principle of semi-smart antennas in load balancing

Compared to fully adaptive antennas that aim an individual beam at each user equipment (UE), the semi-smart antennas approach produces a flexible broadly-shaped coverage pattern for an area and the cost is much lower [16].

\section{SC Allocation Model}

The interference analysed in this paper is in an LTE OFDMA downlink transmission.

It is assumed there are $N$ BSs in the network and in each cell there are $I$ users and $M$ SCs. The $i^{\text {th }}$ user of cell $n$ is denoted by $c_{n, i}$.

The SINR of user $c_{n, i}$ on $S C_{m}$ can be represented by (1):

$$
\operatorname{SINR}_{n, i}(\mathrm{~m})=\frac{P_{\text {signal }}}{P_{n o i s e}+P_{\text {interference }}}=\frac{h_{n, i}^{n}(\mathrm{~m})^{*} p_{n, i}(\mathrm{~m})}{N_{0}(\mathrm{~m})+\sum_{n^{\prime} \neq n, n^{\prime} \in N} \sum_{i \in I} h_{n^{\prime}, i}^{n}(\mathrm{~m})^{*} p_{n^{\prime}, i}(\mathrm{~m})}
$$

where $h_{n, i}^{n}(\mathrm{~m})$ is the channel gain of $B S_{n}$ to user $c_{n, i}$ on $S C_{m}$; $p_{n, i}(\mathrm{~m})$ is the transmission power to user $c_{n, i}$ on $S C_{m} . p_{n, i}(\mathrm{~m})$ has two possible values, shown in (2):

$$
p_{n, i}(\mathrm{~m})=\left\{\begin{array}{c}
p, \text { if } c_{n, i} \text { uses } S C_{m} \\
0, \text { if } c_{n, i} \text { not use } S C_{m}
\end{array} \quad \forall m \in M\right.
$$

This paper uses the SC allocation method proposed in [14] which used a non-cooperative game to maximize the number of users whose QoS requirements are achieved. As a player in game theory, each BS aims to achieve the best SC allocation scheme to maximize its number of qualified users while competing against that of other cells. The end of the game is when no cell can unilaterally increase its utility without any other cell changing its decision.

In a real network, it is not realistic to expect cell boundaries to change rapidly as this would lead to increased handovers and signalling. The approach envisaged is that the cell coverage would be optimized when necessary with changing user distribution. In this paper, the intention is to determine the benefit of using optimal antenna patterns before applying the SC algorithm. A Genetic Algorithm (GA) search algorithm is used to learn the near-optimal antenna coverage pattern; although the GA is slow, it is well known that GA methods lead to near-optimal solutions and hence can be a good benchmark for comparison results.

\section{Overall Design of System Simulator}

The flow chart of the overall system simulator is shown in Figure 3. The overall simulation can be divided into two parts, the network simulator part and the GA part. Previous work [14] does not consider adaptive antenna pattern as an optimization mechanism. In this research, by controlling the semi-smart antennas, GA is able to manage the network resource allocation to maximize the number of qualified users.

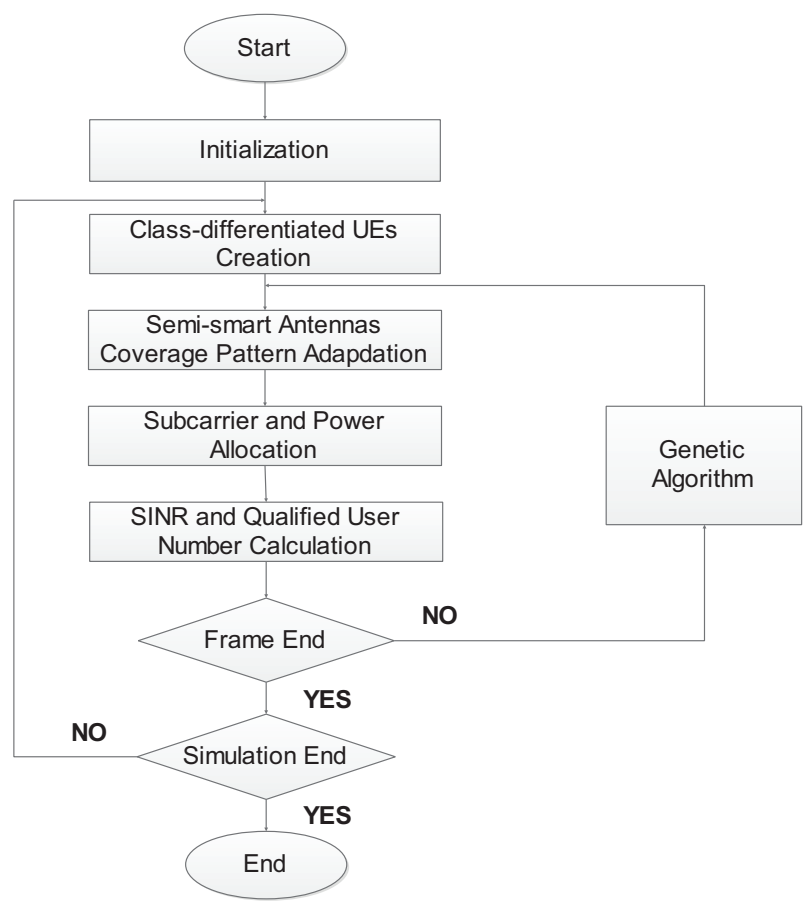

Figure 3 Flow chart of the overall system simulator with GA optimization

\section{THE PROPOSED GA BASED OPTIMIZATION ALGORITHM}

The objective of the proposed scheme is to achieve the maximum number of qualified users. In existing work [14], the antenna pattern is fixed but changing those patterns in 
response to user demand gives extra freedom for optimizing the radio resource. How to do that optimization is an NP-hard problem and GA is an effective tool to solve such a problem due to the way it works with a population of solutions instead of a single solution; GAs do not require any auxiliary information except the objective function values and they use probabilistic rules to guide their search [17].

GAs are search and optimization procedures borrowing the concepts of natural genetics and natural selection to construct search algorithms that are robust and require minimal information about the problem [17]. GAs are well known and described in the literature so the basic operation is not considered in detail here.

\section{1) Solution representation}

The encoding scheme to represent the antenna patterns as chromosomes uses a gain vector $\boldsymbol{G}=\left[\boldsymbol{g}_{1}, \boldsymbol{g}_{2}, \ldots, \boldsymbol{g}_{j}\right]$, in which each gain value is coded as a gene, representing antenna gains along $J$ directions. This determines one antenna coverage pattern. The multi-cell system has $N$ BSs, so that a chromosome is represented as $\left[\boldsymbol{G}_{1}, \boldsymbol{G}_{2}, \ldots \boldsymbol{G}_{N}\right]$. Each gene is mapped to the corresponding antenna element gain (gene values 0 to 20 [16] corresponding to the minimum to maximum coverage).

\section{2) Fitness assignment}

The objective function here is to maximize the qualified user ratio based on the non-cooperative game SC allocation. So for each solution antenna pattern within the GA evolution process, the SC allocation algorithm then works on finding the Nash Equilibrium so that the GA adapts the antenna pattern produced by the semi-smart controllable antenna elements to achieve the objective function.

\section{3) Genetic operators}

In the reproduction operation, roulette selection is applied. Equal single-point crossover operator and fixed mutation operator are used in the crossover operation and mutation operation respectively [17].

\section{Simulation SET-UP AND RESUlts}

\section{A. Simulation Parameters}

Table 1 System parameters for simulation

\begin{tabular}{|l|l|}
\hline Parameter & Value \\
\hline Network topology & 7 hexagonal cells with wrap-around \\
\hline Inter-cell distance & $1 \mathrm{~km}$ \\
\hline Carrier frequency & $2.0 \mathrm{GHz}$ \\
\hline Subcarrier bandwidth & $10 \mathrm{kHz}$ \\
\hline Downlink subcarrier number & 300 \\
\hline Antenna type & $\begin{array}{l}3 \text { sectors semi-smart antennas, with } 6 \\
\text { controllable elements }(0-20 \mathrm{dBi}) \text { for } \\
\text { each cell }\end{array}$ \\
\hline BS antenna height & 75 m \\
\hline Path loss model (dB) & $\begin{array}{l}\text { Hata-Okumura model for an urban } \\
\text { environment }\end{array}$ \\
\hline
\end{tabular}

The system parameters and the parameters for the GA simulation are shown in Tables 1 and 2 respectively. The values chosen in Table 1 follow those in [16].
Table 2 GA evolution parameters

\begin{tabular}{|l|l|}
\hline Evolution Parameter & Value \\
\hline Population size & 50 \\
\hline Generation number & 200 \\
\hline Crossover probability pc & 0.8 \\
\hline Mutation probability pm & 0.1 \\
\hline
\end{tabular}

\section{B. Simulation Results}

Figure 4 shows the convergence of qualified user ratio within one GA antenna pattern solution to validate the credibility of the SC allocation implementation. The qualified user ratio curve in Figure 4 is similar to the result in [14] (Fig.9 in that paper). Different system parameters and user classifications may account for the differences in the actual values between Figure 4 and that in [14].

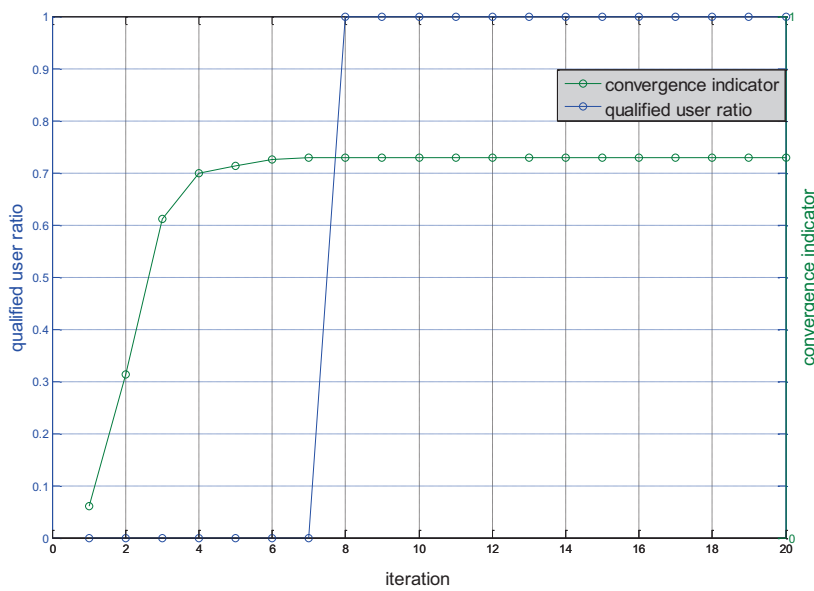

Figure 4 Qualified user ratio and convergency against iteration

One example of the GA evolution on maximizing the qualified user ratio over 200 generations is shown in Figure 5. The scale is exaggerated but it can be seen that the GA functions properly to increase the fitness value as the number of generation increases.

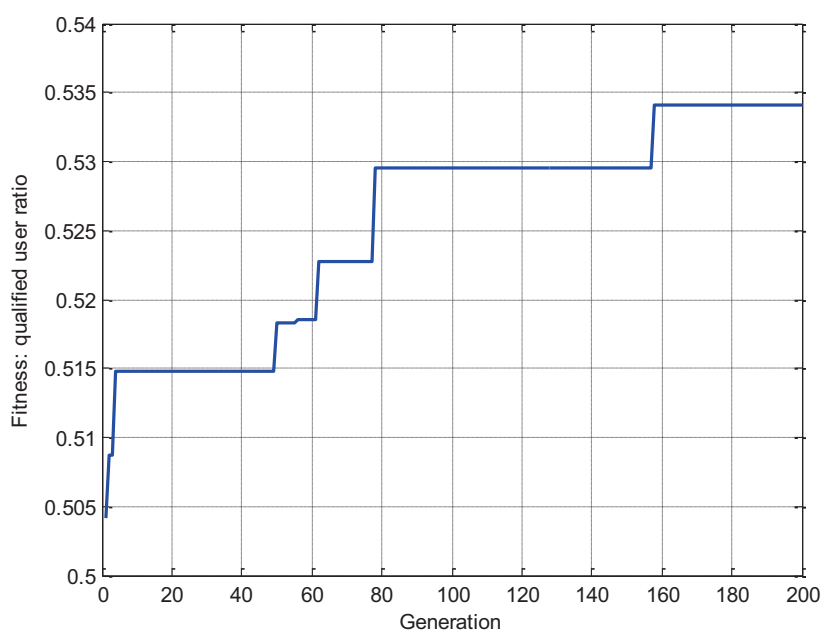

Figure 5 GA evolution on maximizing qualified user ratio 
Figure 6 illustrates the improvement in qualified user number (of around 10\%) from 5 runs when using the GA based adaptive pattern compared with a uniform fixed pattern. This is a significant increase in capacity.

The user number within the 7-cell system varies from 300 to 900 . The desired bit rate for each user is set at $384 \mathrm{kbps}$ [15]. Hotspots at cell centers are used in this research as the user distribution. The GA adaptive cell boundaries have most effects in this kind of load configuration because the optimisation concentrates on improving the performance for the relative small proportion of cell-edge users, The QU ratio sees significant benefits in this user distribution. The internal SC allocation [14] is validated shown in Figure 4.

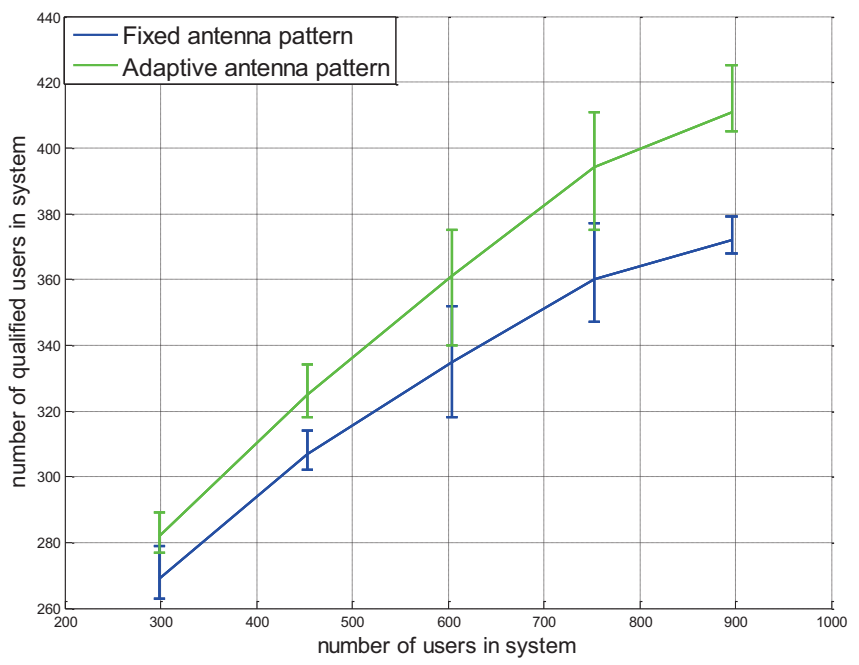

Figure 6 GA improvement in different load networks

One example of the adaptive coverage pattern after 200 generations of GA evolution with 762 UEs is shown in Figure 7. It shows how the GA learns the cell boundaries to achieve the objective of maximizing the number of qualified users.

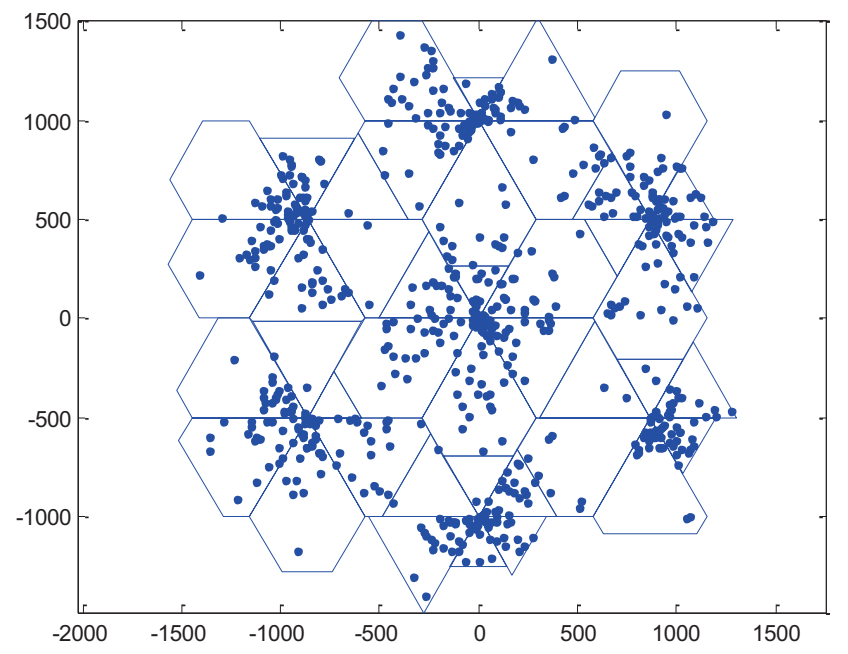

Figure 7 Adaptive coverage pattern after GA evolution

\section{Performance on Qualified Throughput}

Figure 8 shows qualified throughput performance against load. The conclusion from these results is that the overall useful throughput is the same whether an adaptive or fixed antenna is used - however, in an overall view of Figure 6 and Figure 8 , the increase in the number of qualified users demonstrates that the SCs are being used more effectively with the adaptive scenario.

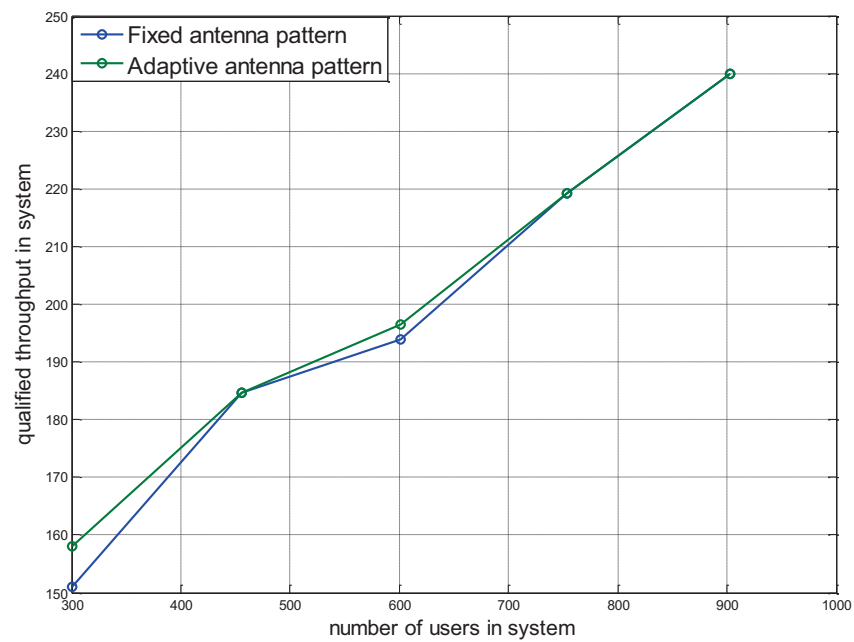

Figure 8 Qualified throughput performance in different load networks

\section{Fixed PU Percentage}

In this section a heterogeneous user class model is applied, with two scenarios considered in this research: 1. Fixed PU percentage; 2. Fixed PU number. With a PU percentage $=0.3$, Figure 9 shows the GA optimization with different objectives: (i) Maximize the qualified user ratio; (ii) Maximize the PU's qualified ratio.

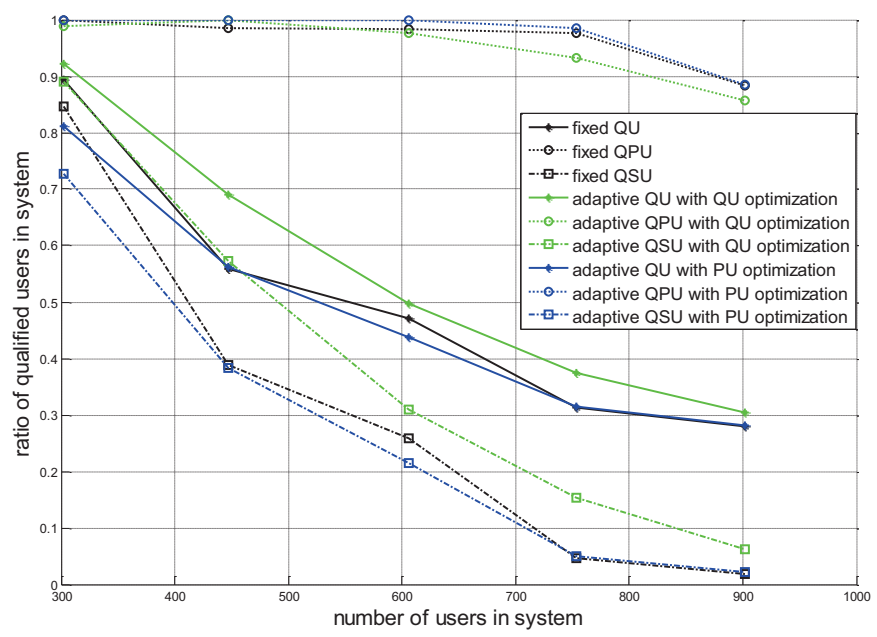

Figure 9 GA improvement over different objectives with PU percentage $=0.3$

The black lines are the qualified user ratios (star for all users; circle for PU, square for SU) with a fixed antenna pattern. This acts as a reference. The green lines are the qualified user ratios with GA optimizing for all users. The blue 
lines are the qualified user ratios with the GA optimizing the PU's qualified ratio.

With GA optimizing the all-user ratio, the QU ratio improves by approximately $10 \%$, the qualified secondary users (QSUs) ratio improves, while the qualified primary users (QPUs) radio may slightly drop. The improvement in QSU probably accounts for the overall QU ratio improvement.

With the GA optimizing the PU's qualified ratio, when the system is not overloaded, the GA will ensure the QPU ratio reaches 1; when the system has excessive users, the GA will slightly enhance the QPU ratio.

In some samples, the reason why QU and QSU ratios are lower than those in with a fixed antenna pattern is that the system is concentrating on protecting the QPU ratio.

\section{E. Fixed PU Number}

In this experiment the PU number is set to be 50, no matter how the system load changes. Figure 10 shows the results from this scenario.

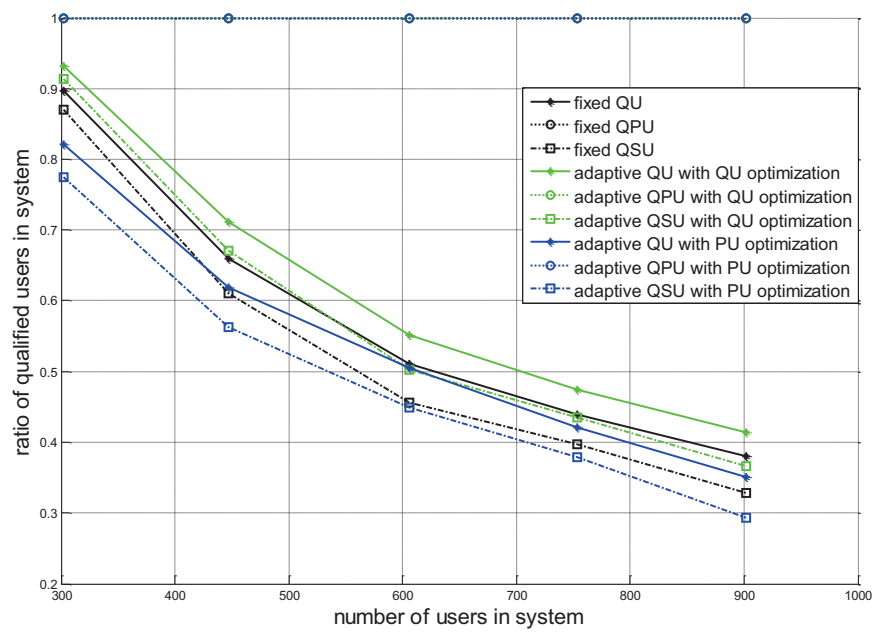

Figure $10 \mathrm{GA}$ improvement over different objectives with PU number $=50$

In fixed PU number scenario, the QPU ratio always reaches 1 because the system always has sufficient resources to ensure that the PU's expected QoS to be achieved. Using GA with the objective of increasing overall QU ratio again brings an improvement of about $10 \%$ over the fixed antenna pattern scenario while keeping the PU's qualified ratio at 1 .

\section{CONCLUSIONS}

In this paper, a novel semi-smart antenna resource management scheme is proposed for QoS-aware multi-cell heterogeneous OFDMA networks. It is demonstrated that using a GA to optimize the BSs' coverage pattern to maximize the number of qualified users does lead to a significant improvement. The QoS requirements across the whole network, especially those of cell edge users, are considered when adjusting the semi-smart antenna gains. Simulation results show that the use of semi-smart antennas improves the number of qualified users by about $10 \%$. With the use of a user class differentiated model, the GA with the increasing QU ratio objective still brings about an improvement of $10 \%$. It also shows that the qualified throughput is as good as that with fixed antennas, showing that the change in antenna patterns reduces interference hence allowing the same number of SCs to be allocated to more users.

\section{REFERENCES}

[1] M. Salem, A. Adinoyi, H. Yanikomeroglu and D. Falconer, "Opportunities and Challenges in OFDMA-Based Cellular Relay Networks: A Radio Resource Management Perspective," IEEE Transactions on Vehicular Technology, vol. 59, no. 5, Jun. 2010.

[2] Y. K. Xiang, "Inter-cell Interference Mitigation through Flexible Resource Reuse in OFDMA based Communication Networks," in European Wireless, Apr. 2007.

[3] H. Kwon and B. Lee, "Distributed Resource Allocation through Non cooperative Game Approach in Multi-cell OFDMA Systems," in IEEE International Conf. Comms., pp. 4345-4350, Jun. 2006

[4] K. Kim, Y. Han and S. Kim, "Joint Subcarrier and Power Allocation in Uplink OFDMA Systems," IEEE Comms. Letter, vol. 9, issue. 6, pp. 526-528, Jun. 2005.

[5] K. Seong, M. Mohseni, and John M. Cioffi, "Optimal Resource Allocation for OFDMA Downlink Systems," IEEE Int'l Symposium on Information Theory, pp. 1394-1398, Jul. 2006.

[6] C. Bae and D. Cho, "Fairness-Aware Adaptive Resource Allocation Scheme in Multihop OFDMA Systems," IEEE Comms. Letter, vol.11, issue. 2, pp. 134-136, Feb. 2007.

[7] M. Kaneko and P. Popovski, "Radio Resource Allocation Algorithm for Relay-aided Cellular OFDMA System," in IEEE Int'l Conf. on Comms., pp. 4831-4836, Jun. 2007.

[8] L. Huang, M. Rong, L. Wang, Y. Xue and E. Schulz, "Resource Allocation for OFDMA Based Relay Enhanced Cellular Networks," in IEEE 65th Vehicular Tech. Conf., pp. 3160-3164, Apr. 2007.

[9] H. Kwon and B. Lee, "Distributed Resource Allocation through Noncooperative Game Approach in Multi-cell OFDMA Systems," in IEEE Int'l Conf. on Comms., pp. 4345-4350, Jun. 2006.

[10] G. Li and H. Liu, "Downlink Radio Resource Allocation for Multi-Cell OFDMA System," IEEE Trans. on Wireless Comms, vol.12, pp. 34513459, Dec. 2006.

[11] C. Koutsimanis and G. Fodor, "A Dynamic Resource Allocation Scheme for Guaranteed Bit Rate Services in OFDMA Networks," in IEEE Int'l Conf. on Comms., pp. 2524-2530, May 2008.

[12] H. Zhu, J. Zhu and K. J. R. Liu, "Non-Cooperative Resource Competition Game by Virtual Referee in Multi-Cell OFDMA Networks," IEEE Journal on Selected Areas in Comms., vol.25, pp. 1079-1090, Aug. 2007.

[13] J. Kim and D. Cho, "Resource Allocation Scheme for Minimizing Power Consumption in OFDMA Cellular Systems," in IEEE 6th Vehicular Tech. Conf., pp. 1862-1866, Oct. 2007.

[14] Y. Liu, L. Cuthbert, X. Yang and Y. Wang, "QoS-Aware Radio Resource Allocation for Multi-Cell OFDMA Network," in IEEE International Conference on Communication Systems (ICCS), Nov. 2012.

[15] Y. Liu, L. Cuthbert, X. Yang and Y. Wang, "QoS-aware Resource Allocation for Multimedia Users in a Multi-cell Spectrum Sharing Radio Network," in 15th ACM Int'l Conf. on Modelling, Analysis and Simualtion of Wireless and Mobile Systems, Aug. 2012.

[16] X. Yang, Y. Wang, D. Zhang and L. Cuthbert, "Resource Allocation in LTE OFDMA Systems using Genetic Algorithm and Semi-Smart Antennas," in IEEE Wireless Communications and Networking Conference (WCNC), Apr. 2010.

[17] K. Deb, "An Introduction to Genetic Algorithms, " in Sadhana, vol. 24, parts 4 \& 5, pp. 293-315, India, Aug. \& Oct. 1990. 